\title{
Safety and Efficacy of Intravenous Colistin in Neonates With Culture Proven Sepsis
}

\author{
Kadir Serafettin Tekgunduz ${ }^{1,{ }^{*}}$; Mustafa Kara ${ }^{1}$; Ibrahim Caner $^{1}$; Yasar Demirelli $^{1}$ \\ ${ }^{1}$ Division of Neonatology, Faculty of Medicine, Ataturk University, Erzurum, Turkey \\ ${ }^{*}$ Corresponding author: Kadir Serafettin Tekgunduz, Division of Neonatology, Faculty of Medicine, Ataturk University, Erzurum, Turkey. Tel: +90-4423446990, Fax: +90-4423447696, \\ E-mail:k.tekgunduz@yahoo.com.tr
}

Received: February 4, 2015; Accepted: February 28, 2015

\begin{abstract}
Background: Although it is well described among adults, intravenous colistin use and its associated toxicities in newborns are poorly understood.

Objectives: We present our experience of efficacy and safety of intravenous colistin in the treatment of sepsis in term and preterm neonates.

Patients and Methods: The records of neonates who received colistin between January 2013 and February 2014 were retrospectively reviewed. All neonates with culture proven nosocomial infections due to multidrug resistant organisms and treated continuously with colistin for more than 72 hours were included in the study.

Results: Patients were evaluated for clinical and microbiological response to the drug and its and side effects. Twelve newborn infants with mean $31.8 \pm 3.5$ weeks gestational age and median $1482(810-3200)$ gram birth weight were included. 11/12 (91.7\%) patients showed microbiological clearance with intravenous colistin. One patient who had recurrent cerebrospinal fluid positive culture was treated with intraventricular colistin. The major side effects observed was hyponatremia and hypokalemia in $2(16.6 \%)$ patients, all infants required magnesium supplementation.

Conclusions: Intravenous colistin administration appears to be safe and efficacious for multidrug-resistant gram-negative infections in neonates, including preterm infants. However, we believe that large prospective controlled studies are needed to confirm its efficacy and safety in neonates.
\end{abstract}

Keywords: Sepsis; Multidrug Resistance; Preterm Infant; Neonate; Colistin; Infection

\section{Background}

Nosocomial infections represent a major problem in neonatal intensive care units (NICUs). Broad-spectrum antibiotics are commonly used since early diagnosis and treatment is crucial. However, this situation leads to an increase in multidrug resistant (MDR) microorganisms (1-4). Especially, MDR gram-negative bacterial infections appear as a significant cause of mortality and morbidity in NICUs. The number of antibiotic options for the treatment of these infections is limited $(1,2)$. The incidence of nosocomial infections in NICUs range between 7\% - 24\% (3, 4). Acinetobacter baumannii, Klebsiella pneumoniae, Pseudomonas aeruginosa and Escherichia coli are considered as the most frequent gram-negative agents (3). At the same time, increasing rates of carbapenem resistance in MDR gram-negative bacteria appears as a major problem. In recent years, physicians have started to use colistin, which is originally an old preparate, to treat nosocomial infections due to MDR gram-negative bacteria (4).

Studies on parenteral use of colistin date back to 1970 . However, as only high dose intramuscular injection was possible in those years, higher rates of nephrotoxicity and neurotoxicity have been reported (5). After intravenous colistin treatment has become a common practice for the treatment of critical patients with MDR gram-negative infections after 2000s, researchers have realized that colistin toxicity is not as high as initially considered and nephrotoxicity is usually reversible (6-11). On the other hand, there is limited data on the effectiveness and safety of colistin in newborns and premature infants (1, 9-11).

\section{Objectives}

The aim of the present report is to present our experience on the effectiveness and safety of intravenous colistin treatment for MDR gram-negative bacterial infections in the NICU of Ataturk University Medical Faculty.

\section{Patients and Methods}

The NICU at our hospital is a tertiary intensive care center with 50 bed capacity. Twelve infants who received intravenous colistin treatment for MDR gram-negative bacterial infections (all isolated microorganisms were

Copyright (C) 2015, Growth \& Development Research Center. This is an open-access article distributed under the terms of the Creative Commons Attribution-NonCommercial 4.0 International License (http://creativecommons.org/licenses/by-nc/4.0/) which permits copy and redistribute the material just in noncommercial usages, provided the original work is properly cited. 
resistant to aminoglycosides, quinolones, penicillins, all cephalosporins and inhibitor combinations, monobactams, and carbapenems) between January 2013 and February 2014 were retrospectively evaluated. The retrospective study was approved by the ethics committee of Faculty of Medicine of Ataturk University.

Standard definitions of center for disease control and prevention (CDC) were used for nosocomial infection (12). The definition of and diagnostic criteria for ventilator-associated pneumonia (VAP) were identical with those of the CDC for infants less than 1 year of age (12): the time of mechanical ventilation $>48$ hours, new or persistent infiltrations on chest X-ray, worsening gas exchange and at least three of the following: (a) temperature instability with no other recognized cause, (b) new onset of purulent sputum, (c) increase in respiratory secretions or increased need for suctioning, (d) WBC $<4,000 / \mathrm{mm}^{3}$ or $>15,000 / \mathrm{mm}^{3}$, (e) respiratory signs (apnea, tachypnea, nasal flaring, retraction, wheezing, rales, or ronchi) and bradycardia ( $<100$ beats/min) or tachycardia $(>170$ beats/min). Severe sepsis was defined as sepsis with organ dysfunction, hypoperfusion or hypotension. Infants with gestational age less than 37 week were considered to be premature.

Multidrug resistant (MDR) gram-negative bacteria were isolated from catheter, cerebrospinal fluid (CSF), urine, tracheal aspirate and blood samples which were sent to the microbiology laboratory in the hospital and patients who received intravenous colistin treatment for minimum 72 hours were included in the study. Prior to intravenous colistin treatment, blood, CSF, and urine cultures from all patients were collected for analysis. For all patients, diagnosis, demographic features, source of resistance microorganism isolation, isolated microorganism, age at onset of intravenous colistin treatment, other route of colistin administration, duration of treatment, and outcome were recorded. Before starting the colistin treatment informed consent was obtained from all parents.

BACTEC 9240 (Becton-Dickinson, NJ) automated system was used to isolate microorganisms from blood and CSF. Positive cultures and samples for which this system could not be used (catheter, tracheal aspirate, urine) were inoculated on chocolate agar, blood agar (5\% sheep blood) and eosin-methylene blue agar. Gram staining was performed on positive strains which were visible after 24 hours to isolate gram-negative bacterial strains. Strain identification and antibiotic susceptibility tests were performed by using VITEK 2 GN and AST-N090 (bioMérieux, Marcy l'Etoile, France) cards. Microorganisms showing resistance to at least three different antibiotic classes were defined as MDR.

All patients received intravenous colistin $(5 \mathrm{mg} / \mathrm{kg} /$ day, three times; colimycin (colistimethate sodium; Kocak Farma, Istanbul, Turkey). Intraventricular colistin was administered in a single dose (10 $\mathrm{mg} /$ day). To evaluate side effects, kidney function tests (creatinine, blood urea nitrogen) and serum electrolyte levels (sodium, calcium, mag- nesium, and potassium) were recorded before and after the treatment. An increase of $>0.5 \mathrm{mg} / \mathrm{dL}$ in serum creatinine levels within 24 hours was considered as acute kidney failure. Presence of apnea and convulsion were monitored to evaluate the neurotoxic effects of intravenous colistin. Antibiotic treatments that were used together with intravenous colistin were recorded. Treatment effectiveness was evaluated according to microorganism growth in control cultures (blood, cathater, CSF, and tracheal aspirate) taken at least 3 day after intravenous colistin treatment, together with clinical and radiographic improvements.

\section{Results}

Twelve patients ( 8 girls, 4 boys) were included in the study. $1(8.3 \%)$ patient was term, while $11(91.7 \%)$ were premature. Mean gestational age was $31.8 \pm 3.5$ weeks and median birth weight was 1482 gram ( $810-3,200) .6$ (50\%) patients underwent major surgical intervention for congenital anomalies (cystic adenomatoid malformation, esophagus atresia, diaphragmatic hernia, CHARGE syndrome and hydrocephalus) and necrotizing enterocholitis (NEC). Among the remaining 6 patients, 1 (8.3\%) patient was a term infant diagnosed with tricuspid atresia. $1(8.3 \%)$ patient was premature and diagnosed with Williams syndrome. The remaining 4 (33.3\%) patients were premature infants with a gestational age of 32 week and a birth weight of $<1,500$ gram.

Patients' clinical features and isolated microorganisms are presented in Table 1. K. pneumoniae was isolated from blood cultures of 3 (25\%) patients and tracheal aspirate cultures of 3 (25\%) patients; A. baumannii was isolated from blood cultures of 1 ( $8.3 \%$ ) patient, tracheal aspirate cultures of $1(8.3 \%)$ patient, urine culture of $1(8.3 \%)$ patient and both blood and CSF cultures of 1 ( $8.3 \%)$ patient; $P$. aeruginosa was isolated from blood cultures of 2 (16.6 $\%)$ patients. All isolated microorganisms were resistant to aminoglycosides, quinolones, penicillins, all cephalosporins and inhibitor combinations, monobactams, and carbapenems. Using clinical and radiographic features, 6 (50\%) patients were diagnosed with severe sepsis, 4 (33.3\%) patients with VAP, $1(8.3 \%)$ patient with urinary tract infection and severe sepsis and 1 (8.3\%) patient with meningitis and severe sepsis (Table 1 ). In addition to the intravenous colistin, despite the resistance and treatment failure, all patients continued to receive their previous antibiotics (meropenem, ciprofloxacin, amikacin) (Table 2). Averagely on postnatal day $34 \pm 16.6$, intravenous colistin ( $5 \mathrm{mg} / \mathrm{kg} /$ day, 3 doses) treatment was started. Control cultures were collected on day 4.5 (3 - 5 days) after treatment. MDR gram-negative bacterial infection persisted in CSF culture of only 1 (8.3\%) patient who had hydrocephalus. This patient was operated for an abscess which was detected during magnetic resonance imaging. Afterwards, external ventricular drainage was performed and the patient received intraventricular colistin for 21 day in addition to intravenous colistin. 
Tekgunduz KS et al.

\begin{tabular}{|c|c|c|c|c|c|c|c|}
\hline Patient & $\begin{array}{l}\text { Gestational } \\
\text { Age, wk }\end{array}$ & Primary Disease & $\begin{array}{c}\text { Age at Onset of } \\
\text { Intravenous Colistin } \\
\text { Treatment (Postna- } \\
\text { tal Days) }\end{array}$ & $\begin{array}{l}\text { Duration of } \\
\text { Intravenous } \\
\text { Colistin } \\
\text { Treatment, d }\end{array}$ & $\begin{array}{l}\text { Source of Micro- } \\
\text { organism }\end{array}$ & $\begin{array}{l}\text { Isolated Micro- } \\
\text { organism }\end{array}$ & Outcome \\
\hline 1 & 36 & prem, CAM & 18 & 26 & TA & K. pneumoniae & Ex \\
\hline 2 & 33 & $\mathrm{DH}$, prem, VAP & 15 & 9 & $\mathrm{TA}$ & A. baumannii & Ex \\
\hline 3 & 31 & prem, sepsis & 26 & 12 & Urine & A. baumannii & $\mathrm{D} / \mathrm{C}$ \\
\hline 4 & 40 & $\begin{array}{l}\text { tricuspid atresia, } \\
\text { sepsis }\end{array}$ & 13 & 12 & TA & K. pneumoniae & Ex \\
\hline 5 & 32 & $\begin{array}{l}\text { prem, Charge } \\
\text { syndrome }\end{array}$ & 56 & 18 & Blood & P. aeruginosa & Ex \\
\hline 6 & 33 & $\begin{array}{l}\text { prem, hydro- } \\
\text { cephalus }\end{array}$ & 59 & 25 & CSF-Blood & A. baumannii & $\mathrm{D} / \mathrm{C}$ \\
\hline 7 & 27 & $\begin{array}{l}\text { prem, operated } \\
\text { NEC, sepsis }\end{array}$ & 42 & 9 & $\mathrm{TA}$ & K. pneumoniae & Ex \\
\hline 8 & 33 & $\begin{array}{l}\text { prem, operated } \\
\text { NEC, sepsis }\end{array}$ & 33 & 21 & Blood & K. pneumoniae & $\mathrm{D} / \mathrm{C}$ \\
\hline 9 & 32 & prem, sepsis & 51 & 21 & Blood & P. aeruginosa & $\mathrm{D} / \mathrm{C}$ \\
\hline 10 & 28 & $\begin{array}{l}\text { prem, sepsis, Wil- } \\
\text { liams syndrome }\end{array}$ & 10 & 8 & Blood & K. pneumoniae & Ex \\
\hline 11 & 29 & prem, sepsis & 41 & 21 & Blood & A. baumannii & $\mathrm{D} / \mathrm{C}$ \\
\hline 12 & 28 & prem, sepsis & 45 & 21 & Blood & К. рпеuтопiae & $\mathrm{D} / \mathrm{C}$ \\
\hline
\end{tabular}

a Abbreviations: CAM, cystic adenomatoid malformation; D/C, discharged; DH, diaphragmatic hernia; Ex, exitus; NEC, necrotizing enterocholitis; Prem, premature infant; TA, tracheal aspirate; and VAP, ventilator-associated pneumonia.

Table 2. Received Antibiotics Before and During Colistin Treatment

\begin{tabular}{lc}
\hline Antibiotic & Number of Patients \\
\hline Meropenem & 4 \\
Meropenem + Amikacin & 7 \\
Ciprofloxacin + Amikacin & 1 \\
\hline
\end{tabular}

We used the dose of colistin recommended by the Infectious diseases society of america in its 2004 guidelines, of $10 \mathrm{mg} /$ day (13). There was no growth on CSF culture on day 4 of the treatment. There was no growth in control cultures of other patients and sepsis findings of these patients improved. Microorganism growth was not present in the other 11 (91.7\%) control cultures. Clinical and radiographic findings of these patients also improved intraventricular.

Pretreatment median creatinine level was 0.4 ( 0.2 - 0.9), while median creatinine level during treatment was 0.3 (0.2 - 0.7). There was a significant reduction in serum $\mathrm{Na}$ and $\mathrm{K}$ levels in only 2 (16.6\%) patients (minimum levels, patient 11: $\mathrm{Na}=111 \mathrm{meq} / \mathrm{L}, \mathrm{K}=2.9 \mathrm{meq} / \mathrm{L}$, patient 12: $\mathrm{Na}=114$ meq $/ \mathrm{L}, \mathrm{K}=2.7 \mathrm{meq} / \mathrm{L}$ ). During treatment, the minimum median serum $\mathrm{Mg}$ level was $0.92 \mathrm{mg} / \mathrm{dL}$ (0.5 - 1.2). Other serum electrolyte levels were in normal range. During follow-up, apnea was observed in 4 patients (33.3\%) within the first 3 day after the onset of intravenous colistin treatment. Convulsion was not observed in any patient except for one (8.3\%) hydrocephalus patient, who had a convulsion prior to treatment.

All patients received intravenous colistin treatment in average $16.9 \pm 6.2$ day. One patient received intraventricular colistin treatment for 21 day. 6 (50\%) patients died. Surviving 6 (50\%) patients were discharged on postnatal median day 81 (41-120).

\section{Discussion}

Different clinical studies have shown that the effectiveness of intravenous colistin on newborns and pediatric age group ranges between $72 \%$ - 98\% (10,11, 14-17). Alan et al. (11) evaluated 21 preterm infants, and reported that $13(62 \%)$ infants received intravenous colistin treatment for MDR A. baumannii growth in culture and 8 infants received intravenous colistin empirically. The authors stated that microorganism growth was present in control cultures of 4 (31\%) patients and the treatment was $81 \%$ effective. Jajoo et al. (9) reported that 18 preterm and term infants received intravenous colistin treatment for MDR gram-negative infections. After treatment, microorganism growth was present in control cultures of 4 patients and they found the treatment was $76 \%$ effective. In the present study, 91.7\%(11/12) of the patients were premature. MDR gram-negative microorganism growth was present in all cultures. Control cultures showed microorganism growth only in a CSF culture of a premature infant (8.3\%) who had hydrocephalus and ventriculo-peritoneal 
shunt. The patient received intraventricular colistin for 21 days in addition to intravenous colistin. There was no growth on CSF culture on day 4 of the treatment. There was no growth in control cultures of other patients and sepsis findings of these patients improved. In the present study we found that intravenous colistin was $91.7 \%$ (11/12) effective.

According to the literature, 5\% - 25\% of intravenous colistin crosses into the CSF and this rate does not exceed $25 \%$ even in meningitis patients with disrupted blood-brain barrier $(18,19)$. Efficacy of intrathecal and intraventricular colistin treatments was reported in various studies $(20,21)$. However, in the literature, data regarding intraventricular colistin usage in the neonatal period are very limited. Alaoui et al. (2) reported that ventriculitis and meningitis in a four-day-old term neonate with meningomyelocele were treated successfully with intraventricular colistin. To the best of our knowledge, our patient represents the second newborn infant that was treated with intraventricular colistin. The absence of meningitis in 11 (91.7\%) patients who had negative control cultures could be the underlying reason for high treatment success in the present study. Alan et al. (11) reported that inhaler colistin treatment led to recovery in case of a patient who had ongoing microorganism growth in endotracheal aspirate culture. The source of microorganism growth, therefore the distribution of intravenous colistin throughout the body should be considered when evaluating effectiveness in treatment. When needed, other routes of colistin administration (intratracheal, intraventricular, and inhaler) should be used.

Currently, the safe dose of intravenous colistin in newborns and preterm infants is not known. However, according to the multicenter studies in recent years, $2.5-5 \mathrm{mg} / \mathrm{kg} /$ day and $5.4 \pm 0.6 \mathrm{mg} / \mathrm{kg} /$ day doses are safe in the pediatric age group $(22,23)$. Jajoo et al. (9) reported using 50,000 $75,000 \mathrm{IU} / \mathrm{kg} /$ day (1 mg colistimethate sodium = 12,500 IU) in newborns and preterm infants, while Alan et al. (11) reported using $2.5-5 \mathrm{mg} / \mathrm{kg} /$ day. In the present study, intravenous colistin was used in $5 \mathrm{mg} / \mathrm{kg} /$ day dose for all patients. Intraventricular colistin was administered in a single dose of $10 \mathrm{mg}$ /day according to the guideline of infectious Diseases society of america in 2004 (13).

Previous studies have reported that the use of carbapenem, ciprofloxacin, ceftazidime, or aminoglycoside-class antibiotics, together with colistin treatment, produces a synergistic effect (24-26). Similarly, despite the development of resistance to these antibiotics in the present study, the patients continued to receive their previous antibiotics treatment during the colistin therapy (Table 2). These antibiotics were not effective alone according to antibiogram results and the clinical situation of patients before the colistin treatment.

Nephrotoxicity is the most frequent side effect of intravenous colistin (27). According to recent studies, the incidence of kidney function impairment due to intravenous colistin treatment ranges between $7.5 \%-18.6 \%(10,28$, 29). However, it should be considered that intravenous colistin treatment, similar to other nephrotoxic drugs, can lead to conditions including infection, septic shock, and multiple organ failure. Therefore, it is difficult to state the nephrotoxicity rates due to intravenous colistin treatment alone (9). In the present study, increased creatinine levels were not observed in any patient. However, marked hyponatremia and hypokalemia were observed in $2(16.6 \%)$ patients. Electrolyte imbalance in these patients improved with supportive care. At least one $\mathrm{Mg}$ replacement was performed for all patients who received intravenous colistin. Cakir et al. (30) reported that hypokalemia, hypocalcemia, hypomagnesemia and metabolic alkalosis development in a premature infant after intravenous colistin treatment and this clinical condition resembled Bartter-like syndrome. Alan et al. (11) reported that hypomagnesemia persisted despite $\mathrm{Mg}$ replacement and $52 \%$ of the patients required potassium replacement. According to the literature, electrolyte imbalances due to intravenous colistin treatment can be managed with replacement therapies and are reversible $(11,30)$. In the present study, intravenous colistin treatment was not discontinued due to electrolyte imbalance and the patients were followed up with replacement therapy. At the end of the treatment, kidney damage was not observed in any patient.

Previous studies have reported neurological side effects due to intravenous colistin treatment, including peripheral and orofacial paresthesia, visual impairment, vertigo, mental confusion, ataxia and convulsion (31). In the present study, convulsion was not observed except for one (8.3\%) patient who had hydrocephalus and an existing convulsion prior to treatment. Apnea was observed during the first three days of intravenous colistin treatment in $4(33.3 \%)$ patients who had extremely low birth weight and severe sepsis. It is hard to distinguish neurological symptoms in newborns and especially premature infants as they are immature. In addition, because previous diseases can cause similar symptoms, it becomes difficult to gain information on neurological side effects of intravenous colistin in this age group (11). While our patients' symptoms did not appear as side effects of intravenous colistin treatment, we believe that stating whether findings such as convulsion and apnea are due to intravenous colistin treatment or not, will not provide accurate information.

Alan et al. (11) reported that the mortality rate was $4 / 21$ (19\%) and three of them had positive postmortem cultures with A. baumannii. 6 (50\%) patients died in the present study. 2 (16.6\%) of these patients deceased due to congenital heart disease (tricuspid atresia, Wiliams syndrome), 2 (16.6\%) patients due to pulmonary hypoplasia (operated for diaphragmatic hernia, operated for cystic adenomatoid malformation), 1 (8.3\%) patient due to operated NEC and postoperative complications. 1 (8.3\%) CHARGE syndrome patient who was operated for choanal atresia and 
tracheoesophagial fistula died before discharge due to aspiration pneumonia. In case of all deceased patients, microorganism growth was not observed in control cultures after intravenous colistin treatment.

Our study has some limitations. The study was designed retrospectively with lack of a control group. It is only a report of a case series. So, we believe that further randomized controlled studies are needed.

In conclusion, our results suggest that intravenous colistin treatment is effective and safe for the treatment of MDR gram-negative infections in newborns and premature infants with culture proven sepsis. However, pharmacokinetic and pharmacodynamic studies are required to determine the optimal dose in this age group.

\section{Authors' Contributions}

Kadir Serafettin Tekgunduz: design the study, acquisition of data, data analysis/interpretation, drafting of the manuscript, critical revision of the manuscript and approval of the article. Mustafa Kara: acquisition of data, data analysis/interpretation, drafting of the manuscript, critical revision of the manuscript. Ibrahim Caner: drafting of the manuscript, critical revision of the manuscript and approval of the article. Yasar Demirelli: drafting of the manuscript, critical revision of the manuscript, and approval of the article.

\section{References}

1. Celik IH, Demirel G, Tatar Aksoy H, Saygan S, Canpolat FE, Uras N, et al. [Acinetobacter baumannii: an important pathogen with multidrug resistance in newborns]. Mikrobiyol Bul. 2011;45(4):716-22.

2. Alaoui SY, Nejmi SE, Chakir AA, Hmamouchi B, Chlilek A. [Intraventricular colistin use in neonatal meningitis caused by Acinetobacter baumanii]. Ann Fr Anesth Reanim. 2011;30(11):854-5.

3. Karaiskos I, Galani L, Baziaka F, Giamarellou H. Intraventricular and intrathecal colistin as the last therapeutic resort for the treatment of multidrug-resistant and extensively drug-resistant Acinetobacter baumannii ventriculitis and meningitis: a literature review. Int J Antimicrob Agents. 2013;41(6):499-508.

4. Souli M, Galani I, Giamarellou H. Emergence of extensively drugresistant and pandrug-resistant Gram-negative bacilli in Europe. Euro Surveill. 2008;13(47):19045.

5. Koch-Weser J, Sidel VW, Federman EB, Kanarek P, Finer DC, Eaton AE. Adverse effects of sodium colistimethate. Manifestations and specific reaction rates during 317 courses of therapy. Ann Intern Med. 1970;72(6):857-68.

6. Ramasubban S, Majumdar A, Das PS. Safety and efficacy of polymyxin B in multidrug resistant Gram-negative severe sepsis and septic shock. Indian J Crit Care Med. 2008;12(4):153-7.

7. Cheng CY, Sheng WH, Wang JT, Chen YC, Chang SC. Safety and efficacy of intravenous colistin (colistin methanesulphonate) for severe multidrug-resistant Gram-negative bacterial infections. Int J Antimicrob Agents. 2010;35(3):297-300.

8. Durakovic N, Radojcic V, Boban A, Mrsic M, Sertic D, Serventi-Seiwerth R, et al. Efficacy and safety of colistin in the treatment of infections caused by multidrug-resistant Pseudomonas aeruginosa in patients with hematologic malignancy: a matched pair analysis. Intern Med. 2011;50(9):1009-13.

9. Jajoo M, Kumar V, Jain M, Kumari S, Manchanda V. Intravenous colistin administration in neonates. Pediatr Infect Dis J. 2011;30(3):218-21.

10. Iosifidis E, Antachopoulos C, Ioannidou M, Mitroudi M, Sdougka M, Drossou-Agakidou V, et al. Colistin administration to pediatric and neonatal patients. Eur J Pediatr. 2010;169(7):867-74.

11. Alan S, Yildiz D, Erdeve O, Cakir U, Kahvecioglu D, Okulu E, et al. Efficacy and safety of intravenous colistin in preterm infants with nosocomial sepsis caused by Acinetobacter baumannii. Am JPerinatol. 2014;31(12):1079-86.

12. Horan TC, Andrus M, Dudeck MA. CDC/NHSN surveillance definition of health care-associated infection and criteria for specific types of infections in the acute care setting. Am J Infect Control. 2008;36(5):309-32.

13. Tunkel AR, Hartman BJ, Kaplan SL, Kaufman BA, Roos KL, Scheld WM, et al. Practice guidelines for the management of bacterial meningitis. Clin Infect Dis. 2004;39(9):1267-84.

14. Celebi S, Hacimustafaoglu M, Koksal N, Ozkan H, Cetinkaya M. Colistimethate sodium therapy for multidrug-resistant isolates in pediatric patients. Pediatr Int. 2010;52(3):410-4.

15. Rosanova M, Epelbaum C, Noman A, Villasboas M, Alvarez V, Berberian $\mathrm{G}$, et al. Use of colistin in a pediatric burn unit in Argentina. J Burn Care Res. 2009;30(4):612-5.

16. Kapoor K, Jajoo M, Dublish S, Dabas V, Gupta S, Manchanda V. Intravenous colistin for multidrug-resistant gram-negative infections in critically ill pediatric patients. Pediatr Crit Care Med. 2013;14(6):e268-72.

17. Falagas ME, Vouloumanou EK, Rafailidis PI. Systemic colistin use in children without cystic fibrosis: a systematic review of the literature. Int J Antimicrob Agents. 2009;33(6):503 e1-503 e13.

18. Markantonis SL, Markou N, Fousteri M, Sakellaridis N, Karatzas S, Alamanos I, et al. Penetration of colistin into cerebrospinal fluid. Antimicrob Agents Chemother. 2009;53(11):4907-10.

19. Antachopoulos C, Karvanen M, Iosifidis E, Jansson B, Plachouras D, Cars O, et al. Serum and cerebrospinal fluid levels of colistin in pediatric patients. Antimicrob Agents Chemother. 2010;54(9):3985-7.

20. Falagas ME, Bliziotis IA, Tam VH. Intraventricular or intrathecal use of polymyxins in patients with Gram-negative meningitis: a systematic review of the available evidence. Int J Antimicrob Agents. 2007;29(1):9-25.

21. Cascio A, Conti A, Sinardi L, Iaria C, Angileri FF, Stassi G, et al. Postneurosurgical multidrug-resistant Acinetobacter baumannii meningitis successfully treated with intrathecal colistin. A new case and a systematic review of the literature. Int J Infect Dis. 2010;14(7):e572-9.

22. Tamma PD, Newland JG, Pannaraj PS, Metjian TA, Banerjee R, Gerber JS, et al. The use of intravenous colistin among children in the United States: results from a multicenter, case series. Pediatr Infect Dis J. 2013;32(1):17-22.

23. Paksu MS, Paksu S, Karadag A, Sensoy G, Asilioglu N, Yildizdas D, et al. Old agent, new experience: colistin use in the paediatric Intensive Care Unit-a multicentre study. Int J Antimicrob Agents. 2012;40(2):140-4.

24. Gupta S, Govil D, Kakar PN, Prakash O, Arora D, Das S, et al. Colistin and polymyxin B: a re-emergence. Indian J Crit Care Med. 2009;13(2):49-53.

25. Gunderson BW, Ibrahim KH, Hovde LB, Fromm TL, Reed MD, Rotschafer JC. Synergistic activity of colistin and ceftazidime against multiantibiotic-resistant Pseudomonas aeruginosa in an in vitro pharmacodynamic model. Antimicrob Agents Chemother. 2003;47(3):905-9.

26. Petrosillo N, Ioannidou E, Falagas ME. Colistin monotherapy vs. combination therapy: evidence from microbiological, animal and clinical studies. Clin Microbiol Infect. 2008;14(9):816-27.

27. Nation RL, Li J. Colistin in the 21st century. Curr Opin Infect Dis. 2009;22(6):535-43.

28. Michalopoulos AS, Tsiodras S, Rellos K, Mentzelopoulos S, Falagas ME. Colistin treatment in patients with ICU-acquired infections caused by multiresistant Gram-negative bacteria: the renaissance of an old antibiotic. Clin Microbiol Infect. 2005;11(2):115-21.

29. Garnacho-Montero J, Ortiz-Leyba C, Jimenez-Jimenez FJ, BarreroAlmodovar AE, Garcia-Garmendia JL, Bernabeu-Wittel IM, et al. Treatment of multidrug-resistant Acinetobacter baumannii ventilator-associated pneumonia (VAP) with intravenous colistin: a comparison with imipenem-susceptible VAP. Clin Infect Dis. 2003;36(9):1111-8. 


\section{Tekgunduz KS et al.}

30. Cakir U, Alan S, Zeybek C, Erdeve O, Atasay B, Yalcinkaya F, et al. Acquired bartter-like syndrome associated with colistin use in a preterm infant. Ren Fail. 2013;35(3):411-3.
31. Spapen H, Jacobs R, Van Gorp V, Troubleyn J, Honore PM. Renal and neurological side effects of colistin in critically ill patients. Ann Intensive Care. 2011;1(1):14 